\title{
UNA EXPERIENCIA PARA LA ELABORACIÓN DE UN PLAN DE MEJORAMIENTO ${ }^{1}$
}

\author{
Margarita Aravena GAETE ${ }^{2}$ \\ Viana U. Figueroa SOTO ${ }^{3}$ \\ Magdalena Palma del RÍO ${ }^{4}$
}

RESUMEN: En el contexto de una institución que reflexiona y dialoga, la elaboración de la Planificación Estratégica que se operacionaliza a través de un Plan de Desarrollo, es una instancia de desafío que enfrenta a la organización con lo que realmente es y hace y lo que ha declarado -ser y hacer- en la planificación institucional. La brecha que se suscita entre ambas constituye el antecedente fundamental en la articulación del Plan de Mejoramiento, cuya base son los resultados obtenidos de la autoevaluación interna. Este arduo proceso, en una institución de la Defensa, lo es más en razón a la cultura y jerarquía organizacional, donde la gestión participativa de todos los estamentos permite hacerlo.

PALABRAS CLAVES: Autoevaluación institucional. Planificación estratégica. Plan de mejoramiento. Plan de desarrollo.

\section{Introducción}

La creación de un Plan de Mejoramiento surgió de la primera autoevaluación interna que implementó el año 2014 la Academia de Guerra del Ejército de Chile. Esta tarea conlleva una mejora continua en todos sus procesos, variables que permiten optimizar en todas las áreas de Gestión Institucional y Gestión de Docencia.

Para conceptualizar el concepto Plan de Mejoramiento se entiende, como el instrumento que describe objetivos y/o metas a lograr en un corto o mediano plazo, el cual plasma tareas a desarrollar según objetivo planteado, además, incluye los indicadores, recursos y responsabilidades para llevarlo a efecto, cuya finalidad es resolver las debilidades encontradas y de esta forma se asumen compromisos de mejoramiento de la organización (CNA, 2010).

Asimismo, tal como lo plantea la Comisión Nacional de Acreditación (2010). este plan también permite reflexionar sobre nuestros procesos y resultados, otorgando

${ }^{1}$ La revisión del estilo del texto fue preparado por Ivonne Zurita.

${ }^{2}$ Doctora en Educación. UAH - Universidad de Alcalá. Coordinadora técnica y administrativa de Acreditación de la Academia de Guerra del Ejército de Chile. Santiago - Chile. 28801 margara.6819@gmail.com

${ }^{3}$ Doctorado en Planificación e Innovación. UAH - Universidad de Alcalá. Asesora educacional en la Academia de Guerra del Ejército de Chile en el Dpto. de Gestión Docente. Alcalá de Henares - Madrid España. 28801 - vianaulda@gmail.com

${ }^{4}$ Magíster en Gestión y Dirección de Centros de Educativos. UB - Universitat de Barcelona. Asesora educacional en la Academia de Guerra del Ejército de Chile en el Dpto. de Acreditación Institucional. Santiago - Chile.08007 - manenapalma@vtr.net 
una capacidad de autorregulación de las acciones implementadas en función de la calidad y por ende de la gestión, docencia, investigación y extensión de esta institución.

A continuación se describirá la experiencia obtenida -tanto positiva como negativa- y pasos que se desarrollaron a lo largo del presente año para levantar un plan de mejoramientos reales y acordes con las debilidades recogidas plasmados en el primer informe de autoevaluación.

\section{Metodología}

\section{Descripción}

En primera instancia, una vez trianguladas todas las debilidades y fortalezas explicitadas por los agentes participantes (directivos, profesores, estudiantes, asesores educacionales, personal militar administrativo), a través de distintas técnicas de recolección de información (encuestas, grupos de discusión y entrevistas), se procedió a ordenarlas bajo dos criterios: prioridad e importancia. Al respecto, dado el tipo de institución - de la Defensa- la cultura educacional militar podría entenderse como un dialogo más bien de tipo vertical, dada la formación profesional de alumnos y profesores; sin embargo, una de las experiencias que se han obtenido a través de esta autoevaluación, es la de constituirse como una comunidad reflexiva, que a través de las diferentes técnicas aplicadas le ha permitido darse cuenta de la importancia que tiene la evaluación en la mejora docente que los centros educativos llevan a cabo, lo que es una cuestión capital (COROMINAS et. al., 2008), originándose una participación horizontal en la comunicación al interior de la institución docente, provocándose un trabajo en conjunto en aras de optimizar la gestión realizada por el personal que conforma esta organización castrense.

En el criterio de importancia, se detallaron aquellas acciones que fueron consideradas como una primera necesidad para realizar los ajustes en forma inmediata (mensual y semestral). Para ello, se ha considerado además que la diversidad de intereses de los asistentes es una dificultad que se puede salvar si se encuentra un denominador común que los una (COROMINAS et al., 2008), como lo es la acreditación institucional ante la comunidad académica.

\section{Clasificación por áreas y aspectos}


Seguido, ordenadas de acuerdo al criterio de prioridad e importancia, se procedió a clasificarlas en dos grandes áreas: Gestión Institucional y Gestión de Docencia. Luego, cada debilidad se encasilló en un concepto amplio común que englobara dicha debilidades, llamados aspectos según la Comisión Nacional de Acreditación (2010).

Estas dimensiones y áreas fueron extraídas desde la Resolución exenta $\mathrm{N}^{\circ} 01$, que describe los lineamientos de la Comisión Nacional de Acreditación. Art. 17 de la ley 20.129 (CHILE, 2006).

\section{Gestión institucional}

La gestión institucional de una entidad es la forma cómo funciona una institución. Implica el establecimiento de un conjunto de políticas y procedimientos no solo para organizar el trabajo de las personas que lo componen, sino también para emplear los recursos materiales y financieros que posee, en pos de sus propósitos y objetivos. Por lo mismo, tiene relación con la organización y estructura institucional, el sistema de gobierno y la administración de los medios humanos, materiales y económicos. Considera estos aspectos:

Estructura y organización institucional: descripción gráfica del ordenamiento y distribución de las funciones que cada departamento, área o unidad de una organización debe cumplir.

- Sistema de gobierno: es el cómo se relaciona el cuerpo directivo con sus funcionarios o trabajadores. Tiene relación con la toma de decisiones, a nivel central.

- Las normas y procedimientos establecidos para la selección, contratación, evaluación y perfeccionamiento del personal directivo, académico y administrativo de la Institución.

- La planificación, ejecución y control de recursos materiales y financieros de la organización, tiene relación con los mecanismos establecidos para asegurar la permanencia y proyección de la organización.

Implica evaluar la existencia y aplicación eficaz de políticas y procedimientos o mecanismos atingentes a los siguientes estos aspectos y establecer si responden o no a los propósitos institucionales:

a. Necesidades de recursos físicos y materiales para planificar su disponibilidad.

b. Uso eficaz de los recursos físicos y materiales.

c. Actualización y desarrollo de los recursos físicos y materiales. 
d. Obtención, manejo y control de los recursos financieros para asegurar el desarrollo actual y futuro de la organización.

- Mecanismos de diagnóstico, planificación, seguimiento y ajuste de las prioridades: se debe constatar si la organización cuenta con un diagnóstico acerca de las condiciones del medio interno y externo, con efectuar ajustes al plan de desarrollo. Constatar si se dispone de lo siguiente:

a. Establecimiento de prioridades institucionales a mediano y largo plazo.

b. Disposición de mecanismos de verificación del grado de avance hacia las metas establecidas. Según los resultados, se ajustan las metas, acciones y recursos.

c. Verificación del cumplimiento de los propósitos y metas establecidas en las dos áreas del quehacer institucional.

Disponibilidad de información para responder a los requerimientos de la gestión: se evalúa si la organización cuenta con información válida y confiable sobre procesos institucionales.

\section{Docencia de pregrado}

Contempla las políticas y mecanismos institucionales para asegurar la calidad de la formación de pregrado, evaluando el diseño y aprobación de los programas de cursos, su implementación y seguimiento, cuyos resultados incidan para revisar y optimizar el currículo, la organización de los programas, las metodologías empleadas en el aula o en terreno, los recursos humanos y materiales asignados. El aseguramiento de la calidad aborda estos aspectos:

- Diseño y provisión de carreras y programas: evaluación de las decisiones sobre la oferta de carrera, su pertinencia, apertura de sedes, definición y revisión de perfiles de egreso, diseño curricular, asignación

- de recursos, seguimiento de los resultados.

- Proceso de enseñanza aprendizaje: evaluar sistema de admisión, métodos pedagógicos, procedimientos evaluativos, seguimiento de los resultados.

- Dotación académica/docente: evaluación de estrategias de reclutamiento, de procesos de seguimiento, perfeccionamiento, renovación.

- Estudiante: evaluar si la Institución cuenta con mecanismos para abordar la progresión de los alumnos durante su carrera, los servicios que ofrece a los alumnos y seguimiento de los egresados, con el fin de obtener la retroalimentación para la toma de 
decisiones o medidas correctivas y de minimizar las debilidades detectadas en la preparación profesional de los alumnos.

- Utilización de los procesos o resultados de la investigación, con el fin de mejorar la calidad de la docencia impartida.

Esquema $\mathbf{N}^{\circ} 1$ - Áreas mínimas de evaluación

\begin{tabular}{|c|c|}
\hline ÁREAS & ASPECTOS \\
\hline \multirow{6}{*}{$\begin{array}{r}\text { GESTIÓN } \\
\text { INSTITUCIONAL }\end{array}$} & - La estructura y organización institucional. \\
\hline & - $\quad$ El sistema de gobierno. \\
\hline & - Las normas y procedimientos asociados. \\
\hline & $\begin{array}{l}\text { - La planificación, ejecución y control de recursos } \\
\text { materiales y financieros. }\end{array}$ \\
\hline & $\begin{array}{l}\text { - Los mecanismos de diagnóstico, planificación, } \\
\text { seguimiento y ajuste de las prioridades de la Institución. }\end{array}$ \\
\hline & - $\quad$ La disponibilidad de información. \\
\hline \multirow{5}{*}{$\begin{array}{l}\text { DOCENCIA DE } \\
\text { PREGRADO }\end{array}$} & - Diseño y provisión de carreras y programas. \\
\hline & - Proceso de enseñanza. \\
\hline & - Dotación académica/ docente. \\
\hline & $\begin{array}{l}\text { - Estudiantes: progresión, servicios y seguimiento de } \\
\text { egresados. }\end{array}$ \\
\hline & $\begin{array}{l}\text { - Utilización de los procesos o resultados de la } \\
\text { investigación para mejorar. }\end{array}$ \\
\hline
\end{tabular}

Fuente: CNA (2010) en Estudio exploratorio sobre efectos de la acreditación institucional en la calidad de la educación superior en Chile.

\section{Elaboración del plan}

Después de la clasificación de las debilidades tanto por dimensiones como por áreas, se procede a la descripción de cada una de ellas conforme al siguiente formato. 
Esquema $\mathbf{N}^{\circ} 2$ - Modelo de Plan

\begin{tabular}{|c|c|c|c|c|c|c|c|c|c|}
\hline $\begin{array}{c}\text { Dimensión } \\
\text { (Áreas por } \\
\text { Mejorar) }\end{array}$ & \begin{tabular}{|c|} 
Aspecto a \\
mejorar \\
(Objetivos/meta \\
s)
\end{tabular} & $\begin{array}{c}\text { Acciones } \\
\text { para } \\
\text { mejorarlo }\end{array}$ & $\begin{array}{c}\text { Tareas } \\
\text { o } \\
\text { proyectos } \\
\text { jinteredios }\end{array}$ & $\begin{array}{c}\text { Mecanismos } \\
\text { y/o } \\
\text { Indicadores }\end{array}$ & $\begin{array}{c}\text { Unidad } \\
\text { Responsabl } \\
\text { e }\end{array}$ & $\begin{array}{c}\text { Financiamien } \\
\text { to }\end{array}$ & $\begin{array}{l}\text { Plazo } \\
\text { Término }\end{array}$ & $\begin{array}{c}\text { Resultado } \\
\text { esperado } \\
\text { (\% Avance) }\end{array}$ & Observaciones \\
\hline $\begin{array}{l}\text { Sistema de } \\
\text { Gobierno y } \\
\text { Planificación } \\
\text { Estrateogica }\end{array}$ & & & & & & & & & \\
\hline $\begin{array}{l}\text { Gestión de } \\
\text { recursos } \\
\text { humanos }\end{array}$ & & & & & & & & & \\
\hline $\begin{array}{l}\text { Infraestructura } \\
\text { y } \\
\text { equipamiento }\end{array}$ & & & & & & & & & \\
\hline $\begin{array}{l}\text { Gestión de la } \\
\text { Información }\end{array}$ & & & & & & & & & \\
\hline Diseño curricula & & & & & & & & & \\
\hline $\begin{array}{l}\text { Proceso de } \\
\text { enseñanza }\end{array}$ & & & & & & & & & \\
\hline $\begin{array}{l}\text { Evaluación } \\
\text { curricular }\end{array}$ & & & & & & & & & \\
\hline $\begin{array}{l}\text { Evaluación } \\
\text { docente }\end{array}$ & & & & & & & & & \\
\hline Evaluación & & & & & & & & & \\
\hline $\begin{array}{l}\text { Recursos de } \\
\text { apoyo a la } \\
\text { docencia }\end{array}$ & & & & & & & & & \\
\hline $\begin{array}{l}\text { Progresión } \\
\text { de los alumnos }\end{array}$ & & & & & & & & & \\
\hline Extensión & & & & & & & & & \\
\hline
\end{tabular}

Fuente: Chile (2011).

Para un mejor entendimiento de cada aspecto que contiene el Plan de Mejoramiento, se describen a continuación las definiciones conceptuales de cada uno de ellos.

- Dimensión (Área por mejorar): los principales ámbitos que se desprenden de la misión del Instituto y que serán analizados desde la perspectiva de gestión institucional y de docencia.

- Aspecto por mejorar (objetivos/metas): los componentes de un proceso que se determinan y definen para optimizarlos o corregirlos en una gestión integral.

- Acciones para mejorarlo: prácticas definidas e intencionadas con un propósito acotado y factible.

- Tareas o proyectos intermedios: conjunto de actividades que se establecen en forma secuenciada y específica con el fin de alcanzar el objetivo propuesto, articulado con la acción de mejora. 
- Mecanismos y/o indicadores: expresiones numéricas que permiten evidenciar las tareas, de forma medible en cuanto a porcentaje de logro en relación a la meta propuesta.

- Unidad responsable: departamento o sección a cargo a de cumplir con el objetivo propuesto.

- Financiamiento: asignación de una cantidad de dinero que se requiere en una organización para concretar una actividad o proyecto.

- Plazo término: fecha de conclusión.

- Resultado esperado (\% avance): el grado de desarrollo alcanzado asociado a cada una de las áreas conforme a una escala de porcentajes.

- Observaciones: sugerencias o aportes que se detallan como antecedentes para fundamentar una actividad o tarea.

\section{Consenso por áreas}

Una vez terminado el Plan de Mejoramiento, se envía vía correo electrónico a los jefes de áreas, tales como: Jefe de Estudios, Jefe del Centro de Estudios Estratégicos, Jefe del Centro de entrenamiento Operativo Táctico, Jefe de Pregrado, Jefe de Posgrado, Jefes Departamento Gestión Académica, Jefe de Recursos Humanos, Jefe de Finanzas, además de las personas involucradas en la coordinación de la Planificación de Pregrado y Posgrado, con la finalidad que validen y entreguen sus aportes por la misma vía.

\section{Aprobación del Director de la organización}

El plan de mejoramiento debe ser visado por el Director de la institución, con el fin de de comprometer su apoyo a todas las tareas asignadas y en forma paralela determinar las acciones que requieren financiamiento para su ejecución.

\section{Difusión del plan}

Finalmente, el plan ya consensuado por los jefes de áreas y aprobado por el Director, entonces, se debe difundir a todo el personal de la organización, adjuntando un escrito, en el cual se organiza cada grupo de personas que conformarán los comités de trabajos en forma heterogénea para optimizar las acciones complejas y exista gestión participativa, con el fin de que se cumplan los objetivos tranzados, pero estos procesos 
de mejora deben alinearse y ajustarse a la normativa educacional que dispone el Ejército de Chile y la Comisión Nacional de Acreditación.

\section{Evaluación y monitoreo del plan}

Luego de la difusión del Plan, se realizarán capacitaciones en el tema de indicadores al personal responsable de cumplir las diferentes tareas, con la finalidad que identifiquen los avances de los procesos y productos que deben generar para la mejora continua; además, se implementarán talleres prácticos para trabajar en conjunto entre el Depto. de Gestión y los involucrado de la creación y levantamiento de los indicadores de insumos, proceso y de producto, y de esta manera consensuar lo que efectivamente se puede trabajar; como también, lograr que este monitoreo y control sea más amigable y produzca un aprendizaje relajado, significativo para los integrantes de la organización, dado que no resulta fácil aplicar un proceso de seguimiento en una cultura que no está acostumbrada a autoevaluarse internamente.

\section{Esquema $\mathbf{N}^{\circ} 3$ - Evaluación del Plan de Mejoramiento}

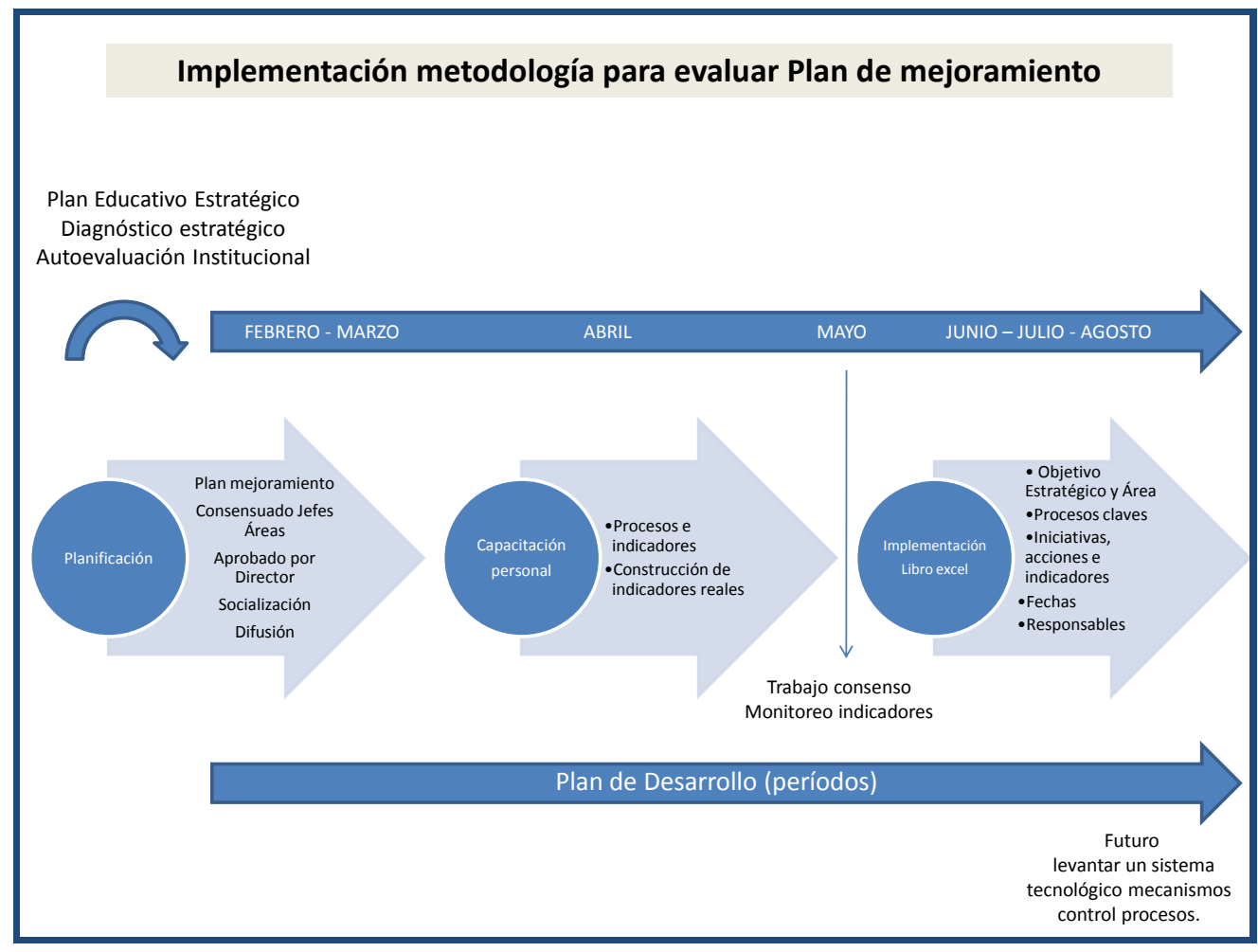

Fuente: Elaboración propia. 


\section{Reflexiones}

Producto de la experiencia en la elaboración del Plan de Mejoramiento aplicado a una entidad del ámbito superior, específicamente en una organización del área de la Defensa, se explicitarán algunas reflexiones con la finalidad de compartir las ventajas y desventajas, que servirán de guía y de retroalimentación para implementar o reproducir esta metodología en otras instituciones educacionales.

El Plan de Mejoramiento es una herramienta útil y que da la oportunidad del mejoramiento continuo de los procesos, actividades, acciones, tareas de cualquier organización, que desee hacer ajustes y optimizaciones en su gestión, tanto a un largo como corto plazo. Además, evidencia que el esfuerzo y los recursos empleados han valido la pena, que se trabaja mejor en equipo, que todos los integrantes de la comunidad pueden ser impulsores de la mejora y la innovación en los centros educativos (COROMINAS et. al., 2008).

Esta metodología se puede reproducir en cualquier entidad educativa, porque es flexible y se puede adaptar de acuerdo a la realidad de cada institución, además, se deben dar las instancias y espacios para que los integrantes de la organización maduren y le encuentren sentido primero a la autoevaluación interna y luego, producto de los resultados del informe de autoevaluación, las debilidades sean trabajadas según importancia y prioridad, de acuerdo a los propósitos que persigue la institución, porque no es fácil a nivel organizacional instaurar la cultura evaluativa; por lo tanto, es necesario dar las instancias de aprendizaje a las personas, ello es lo trascendental.

Respecto a la validación y consenso con los diferentes actores directivos que toman las decisiones en la organización, es importante que revisen el Plan de Mejoramiento antes que el Director de la institución, porque de esta forma se asegura la gestión participativa y, por ende, que cada tarea o acción descrita efectivamente se lleve a efecto, por parte de los subordinados de sus áreas, según corresponda, y finalmente, exista una alineación con todo los estamentos directivos involucrados en esta tarea. 
ABSTRACT: For an institution that carries out an internal self- evaluation, the preparation of a Strategic Plan which is executed through a development plan, represents a challenge that confronts the organization with what they actually do and what has been stated -be and do- in the institutional plan. The breach that rises between them becomes a key element when making an improvement plan, which input is the results from the internal self-evaluation process. This hard process, in an institution of the Defense area, becomes even harder due to an institutional particular culture and organizational hierarchy, where the participation of all the levels involved allows its execution.

KEYWORDS: Institutional self-evaluation. Strategic plan. Improvement plan. Development plan.

\section{REFERENCIAS}

CHILE. Ejército de Chile. División Educación. Comando de Educación y Doctrina. Reglamento Educación Militar RAE-01001. Chile, 2011.

. Ministerio de Educación. Ley n.20.129, 27 de setembro de 2006. Establece un sistema nacional de aseguramiento de la calidad de la educacion superior. 17 nov. 2006. Disponible en:

<https://www.cnachile.cl/Documentos\%20de\%20Paginas/Ley_Aseg_Calidad\%2020.12 9.pdf $>$. Acceso en: 15 abr. 2015.

COMISIÓN NACIONAL DE ACREDITACIÓN [CNA]. Estudio exploratorio sobre efectos de la Acreditación Institucional en la calidad de la educación superior en Chile: informe ejecutivo. Santiago, abr. 2010. Disponible en:

$<$ https://www.cnachile.cl/Biblioteca\%20Documentos\%20de\%20Interes/Estudio\%20IPS OS\%20-\%20Informe\%20Ejecutivo.pdf>. Acceso en: 16 abr. 2015.

COROMINAS CANAL, M. et al. Comunidades reflexivas: observarse para compartir descubrimientos. Cuadernos de Pedagogía, [S.1.], n.379, p.55-58, mayo 2008. 